Revista Latinoamericana de la Papa 22 (2): 55 - 65

ISSN: $1853-4961$

http://www.papaslatinas.org/revista.html

\title{
Efecto de la incorporación de residuos de cosecha de tarwi (Lupinus mutabilis Sweet) en sistemas marginales de producción de papa de regiones andinas semiáridas de Cochabamba, Bolivia
}

\author{
P. Mamani-Rojas ${ }^{1}$, J.J. Calisaya-Vargas ${ }^{2}$
}

Recibido: $22 / 11 / 2018$

Aceptado: $22 / 12 / 2018$

Accesible en línea: Diciembre de 2018

\section{Resumen}

El estudio se llevó a cabo durante las campañas agrícolas 2014-2015 y 20152016, en comunidades del municipio de Anzaldo (Cochabamba), ubicadas a $3040 \mathrm{msnm}$. La precipitación del lugar es de $450 \mathrm{~mm} / \mathrm{año}$ y su temperatura mínima y máxima es de $4^{\circ} \mathrm{C}$ y $23^{\circ} \mathrm{C}$, respectivamente. Sus suelos son de escasa profundidad, baja fertilidad y escasa materia orgánica $(0,9 \%)$. El presente estudio buscó determinar el efecto de la incorporación al suelo de los residuos de cosecha de tarwi, en el sistema de rotación tradicional de cultivos y en la recuperación de los suelos. Esta incorporación logra incrementar la productividad de papa en $51 \%$ y de quinua en $58 \%$. En ambos cultivos, la combinación de rastrojo de tarwi con guano animal, logra incrementar aún más su rendimiento. También se determinó que esta incorporación incrementa la respiración del suelo, lo que sugiere que se genera mayor actividad microbiana debido al incremento de la materia orgánica del suelo (de $0,7 \%$ a $1,03 \%$ ) lo que repercute en un mayor contenido de $\mathrm{N}$ (de $27 \mathrm{ppm}$ a 40 ppm), $\mathrm{P}$ (de 8 ppm a 13 ppm) y K (0,25 a 0,35 meq/100 g de suelo). Por tanto, se sugiere promover la incorporación de los residuos de cosecha de tarwi, para favorecer al sistema de cultivos y la mejora del suelo.

Palabras claves adicionales: Incorporación de residuos, sistemas de cultivos, rotación de cultivos, recuperación de suelos, respiración de suelos.

Effect of the incorporation of tarwi (Lupinus mutabilis Sweet) crop residues in marginal potato production systems of semi-arid Andean regions of Cochabamba, Bolivia

\section{Summary}

Incorporation effect of tarwi (Lupinus mutabilis Sweet) crop residues in crops system and soils recovery of semi-arid Andean regions of Cochabamba. The study was carried out during the agricultural campaigns 2014-2015 and 2015-2016, in communities of the Municipality of Anzaldo (Cochabamba), located at 3040 meters above sea level. In that area, the precipitation is $450 \mathrm{~mm} /$ year and its minimum and maximum temperature is $4^{\circ} \mathrm{C}$ and $23^{\circ} \mathrm{C}$, respectively. Its soils are of low depth, low fertility and scarce organic matter $(0.9 \%)$. The present study sought to determine the effect of tarwi crop residues incorporation in soil, under the traditional crop rotation system and in the recovery of soils. This incorporation increases the potato productivity in $51 \%$ and quinoa in $58 \%$. In both crops, the combination of tarwi stubble with animal guano increases its performance even more. It was also determined that this incorporation increases soil respiration, suggesting 
that greater microbial activity is generated due to the increase of soil organic matter (from $0.7 \%$ to $1.03 \%$ ), affecting a higher content of $\mathrm{N}$ (from $27 \mathrm{ppm}$ to $40 \mathrm{ppm}$ ), $\mathrm{P}$ (from $8 \mathrm{ppm}$ to $13 \mathrm{ppm})$ and $\mathrm{K}(0.25$ to $0.35 \mathrm{meq} / 100 \mathrm{~g}$ of soil $)$. Therefore, it is suggested to promote the incorporation of tarwi crop residues, to favoring the crop system and soil improvement.

Additional keywords: Incorporation of waste, cropping systems, crop rotation, soil recovery, soil respiration.

\section{Introducción}

Las prácticas de manejo del cultivo, tales como la labranza, rotación de cultivos, incorporación de residuos de cosecha, pueden modificar la materia orgánica del suelo (Espinoza et al.2007).

El mantenimiento de la materia orgánica del suelo regula favorablemente el ambiente físico, químico y biológico del suelo (Follet 2001, Spedding et al., 2004). La variación de la fracción orgánica del suelo y la intensidad de laboreo, determinan la condición física del mismo (Eiza et al. 2005).

Estudios en suelos de textura similar, han demostrado que los suelos con alto contenido de materia orgánica, tienen significativamente mayor Capacidad de Agua Disponible que los suelos con menor materia orgánica (Hudson 1994).

Estudios de rotación de cultivos han mostrado efectos positivos sobre el mantenimiento de niveles altos de la materia orgánica del suelo (Campbell et al. 1996, Omayet al. 1997, Espinoza et al.2007). En la rotación de cultivos, las leguminosas juegan un papel importante en las fracciones de la materia orgánica del suelo, debido a su efecto sobre la relación C/N (Potter et al. 1998).

Desde épocas remotas se reconoce al lupino como mejorador de la fertilidad del suelo, fijador de nitrógeno atmosférico y buen extractor de los nutrientes de las capas profundas del suelo, debido a su desarrollo radicular profundo (Barrera 2015). El Lupinus mutabilis se adapta a condiciones de baja precipitación pluvial, suelos de baja fertilidad y a temperaturas entre $-9.5^{\circ} \mathrm{C}$ y $28^{\circ} \mathrm{C}$ (Cifuentes et al. 2001).

Los sistemas de producción de las regiones andinas semiáridas de Cochabamba (Bolivia), se tornan cada vez más vulnerables a las presiones que ejercen los mismos productores sobre sus suelos, obligados por la sobrevivencia y los cambios en el clima, lo cual está alterando los patrones de manejo de los cultivos y del ganado (Oros et al.2018). Los suelos de estas regiones, se caracterizan por su baja profundidad, poca fertilidad, son sensibles a la erosión debido al bajo contenido de materia orgánica como consecuencia de la mínima capacidad de reposición de materia orgánica al suelo, debido a la cada vez menor población ganadera ovina, la cual aporta muy poco guano al sistema y porque los residuos de cosecha de cultivos predominantes, son extraídos para alimentar a los animales o son comercializados para otras necesidades, $\mathrm{y}$ por tanto no son devueltos al suelo.

Estas causas, junto al cambio climático, están agudizando la disminución gradual de la capacidad productiva de los suelos, cuyos principales indicadores, según Fonte y Vanek (2012), son: Bajo contenido de materia orgánica $(0,5 \%$ a $2 \%), \mathrm{pH}$ ácido $(4,5$ a 6,5), bajo contenido de nitrógeno y fósforo, baja capacidad de intercambio catiónico (3 a $10 \mathrm{meq} / 100 \mathrm{~g}$ de suelo) y poca profundidad (15 a 25 $\mathrm{cm})$. 
La correcta elección de una combinación de rotaciones, labranzas e incorporación de residuos, puede ser una herramienta útil para reducir los riesgos de degradación del suelo y para maximizar la producción con el mínimo compromiso para el ambiente. Las interacciones entre los efectos de corto y largo plazo entre rotaciones, labranzas y otras prácticas, son tan estrechas y complejas, que generalmente es difícil separarlos y analizarlos individualmente. Por esta razón, es preferible analizar los sistemas de cultivo más que las prácticas aisladas, ya que este término las incluye junto con sus interacciones, dentro del contexto general del sistema de producción (Studdert y Echeverría 2000).

$\mathrm{Si}$ bien las leguminosas aportan al mejoramiento de la fertilidad de los suelos, los agricultores de la zona no tienen un conocimiento adecuado de esta práctica, debido a que el cultivo del tarwi presenta un desarrollo leñoso, dificultando su incorporación con tracción animal o manual, por lo que los agricultores tienden a quemar o extraer el rastrojo para utilizar como leña. Por otra parte, debido a la tasa de migración elevada y la demanda de mayor cantidad de mano de obra, la agricultura tiende a la mecanización, por lo que la incorporación de residuos de cosecha podría realizarse con maquinaria agrícola.

En este sentido, el objetivo del presente estudio fue determinar el efecto de la incorporación de los residuos de cosecha de tarwi, en el sistema de rotación tradicional de cultivos, y en la recuperación de los suelos de la región alto andina semiárida de Anzaldo (Cochabamba).

\section{Materiales y Métodos}

El estudio se llevó a cabo durante las campañas agrícolas 2014-2015 y 2015-
2016. Los ensayos se establecieron en comunidades del municipio de Anzaldo (Cochabamba-Bolivia), ubicado a $62 \mathrm{~km}$ de la ciudad de Cochabamba, a una altitud de $3040 \mathrm{msnm}$ y en las coordenadas $17^{\circ} 46^{\prime} 46^{\prime}$ " de latitud Sud y 65'55'56" de longitud Oeste. El clima durante el cultivo se caracteriza por una precipitación promedio de $450 \mathrm{~mm} / \mathrm{año} \mathrm{y}$ temperaturas que van de una mínima de $4^{\circ} \mathrm{C}$ a una máxima de $23^{\circ} \mathrm{C}$.

Los suelos predominantes son de escasa profundidad con presencia de una roca dura continúa (barrera física) y mucha pedregosidad, lo cual describe a un suelo de tipo Leptosoles Éutricos con algunos Cambisoles Éutricos y Dístricos en campos con suelos más profundos. Evaluaciones previas de la región (PROINPA 2012), muestran que los suelos son semiácidos $(\mathrm{pH} 5,7)$, de baja Conductividad Eléctrica (0,06 $\mathrm{mmhos} / \mathrm{cm}$ ) es decir no son salinos, muy baja CIC (6,22 meq/100 g) atribuible a su bajo contenido de materia orgánica $(0,93 \%)$, consecuentemente un pobre contenido de $\mathrm{N}(0,06 \%)$. El P también es bajo (4,92ppm) y su K moderado $(0,91$ meq/100 g). En general son suelos Franco Arenosos a Franco Limosos y su densidad aparente es alta (1,51 $\mathrm{g} / \mathrm{cc})$ como producto de su compactación.

El primer año (2014-2015) se implantaron dos ensayos para ver el efecto de la incorporación al suelo, de residuos de cosecha (rastrojo) de tarwi (Lupinus mutabilis), sobre los cultivos de papa (Solanum tuberosum ssp. andigena) y quinua (Chenopodium quinua).

Se utilizaron parcelas donde un año antes (ciclo agrícola 2013-2014) se había cultivado tarwi, cuyos residuos de cosecha fueron incorporados al suelo según definía el tratamiento utilizado. 
Para cada cultivo se utilizó el diseño de Parcelas Divididas en Franjas, en Bloques Completos al Azar, con tres repeticiones.

La unidad experimental consistió de una parcela de $10 \mathrm{~m}$ de largo y $3 \mathrm{~m}$ de ancho.
Dentro de cada unidad experimental se tomaron 3 sub muestras de $5 \mathrm{~m} 2$. La Tabla 1 describe los factores, niveles y tratamientos del estudio.

Tabla 1. Descripción del diseño experimental y los tratamientos utilizados en el ciclo agrícola 2014 - 2015 para los estudios en papa y quinua

Año

Año 2014 - 2015

agrícola

2013 - Factor: "Residuos $\quad$ Factor: $\quad$ Tratamiento

2014 vegetales de "Guano ovino"

tarwi" (Sub parcela)

(Parcela)

\begin{tabular}{llll}
\hline Parcela & Sin incorporación & Sin guano & T1 $=$ Sin incorporación y sin guano \\
con tarwi & Con guano & T2 $=$ Sin incorporación y con guano \\
& Con incorporación & Sin guano & T3 $=$ Con incorporación y sin guano \\
& Con guano & T4 $=$ Con incorporación y con guano
\end{tabular}

Se utilizaron parcelas donde en el ciclo agrícola anterior (2013 - 2014) se cultivó tarwi

Las incorporaciones del rastrojo de tarwi se realizaron después de la cosecha del grano. Para la incorporación, se utilizó tracción mecánica, con rastra de disco para el picado, y arado de disco para su incorporación. El efecto de la incorporación de rastrojo de tarwi se evaluó en los cultivos de papa y quinua en la campaña 2014-2015 y solo papa en la campaña 2015-2016, debido a que es un cultivo importante en el sistema de Anzaldo.

Para la siembra de papa y quinua, la apertura de surcos se realizó con yunta, manteniendo una distancia de $70 \mathrm{~cm}$ entre surcos. La densidad de siembra fue de 12 $\mathrm{kg} / \mathrm{ha}$ de quinua y $32 \mathrm{qq} / \mathrm{ha}$ de papa. El guano ovino se aplicó durante la siembra a razón de 5 t/ha. El aporque y desmalezado se realizaron con yunta, a los 30 días después de la emergencia.

El segundo ciclo agrícola (2015 - 2016), en un sitio diferente, se repitió el ensayo de incorporación de residuos de cosecha de tarwi, para ver esta vez su efecto solo sobre el cultivo de papa. Se utilizaron parcelas donde un año antes (ciclo agrícola 2014-2015), en una parte se 
había sembrado tarwi y otra parte donde se dejó el terreno en descanso. También se utilizó el diseño de Parcelas Divididas en Franjas en Bloques Completos al Azar, con tres repeticiones. La Tabla 2 describe los factores, niveles y tratamientos del estudio.

Tabla 2. Descripción del diseño experimental y los tratamientos utilizados en el ciclo agrícola 2015 - 2016 para el cultivo de papa.

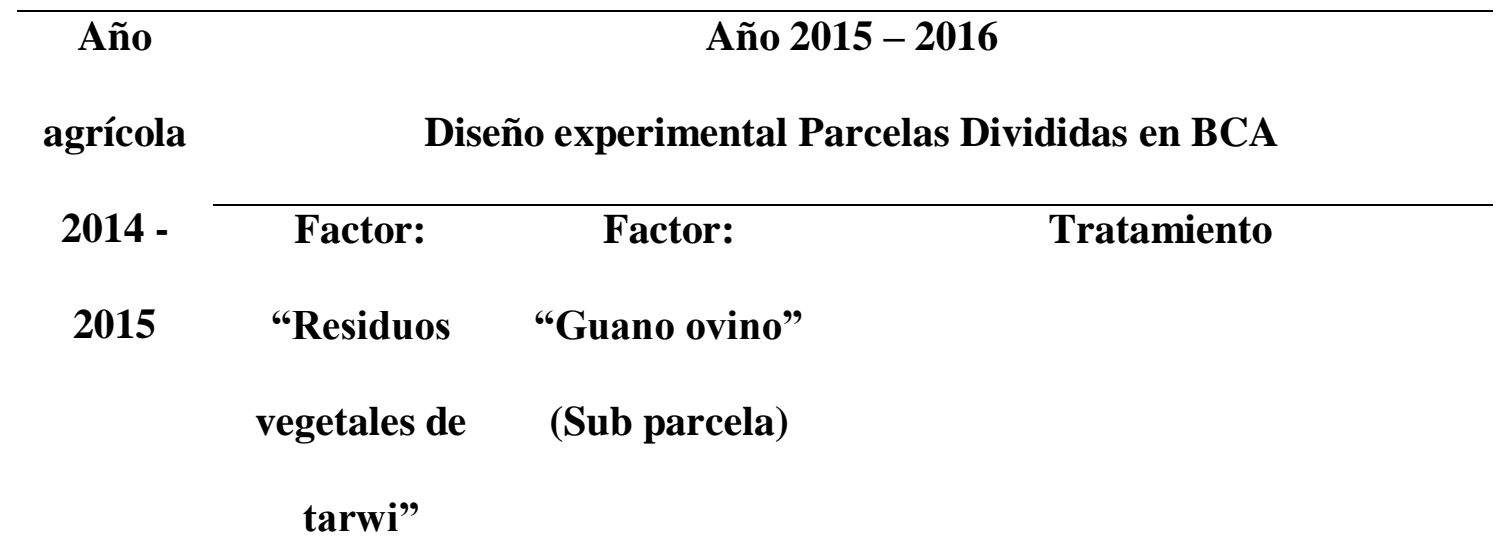

(Parcela)

\begin{tabular}{llll}
\hline Parcela & Con tarwi y sin & Sin guano & T1 $=$ Sin incorporación y sin guano \\
con tarwi & incorporación & Con guano & T2 $=$ Sin incorporación y con guano \\
& Con tarwi y & Sin guano & T3 $=$ Con incorporación y sin guano \\
& con & Con guano & T4 $=$ Con incorporación y con guano \\
Parcela & Sin tarwi y sin & Sin guano & T5 $=$ Sin tarwi y sin guano \\
en & incorporación & Con guano & T6 $=$ Sin tarwi y con guano \\
descanso & & &
\end{tabular}

Se utilizaron parcelas en descanso y parcelas donde en el ciclo agrícola anterior (2014 - 2015) se cultivó tarwi

La incorporación y el manejo del cultivo fueron similares al primer año. En ambos años, las variables de evaluación en los cultivos, fue el rendimiento y en el caso del suelo, la respiración y otras variables de laboratorio ( $\mathrm{K}$ intercambiable, materia orgánica total, $\mathrm{N}$ total y $\mathrm{P}$ disponible), para lo cual se tomaron muestras de suelo durante el desarrollo del cultivo. 


\section{Resultados y discusión}

La Figura 1 muestra el efecto de los residuos de cosecha de tarwi incorporados al suelo (rastrojo de tarwi), sobre los cultivos de papa y quinua, como alternativas en la rotación de cultivos en dos ecoregiones de Anzaldo. Como testigo referencial también se incluye al guano ovino. Las letras mayúsculas comparan las medias del factor "rastrojo de tarwi" (parcela principal) mientras que las letras minúsculas comparan las medias del factor "guano ovino" (sub parcela).

En papa claramente existe un efecto positivo del rastrojo de tarwi, principalmente cuando se acompaña con guano. En quinua, el rastrojo también incrementa su productividad, aún si no va acompañado con guano. En ambos cultivos, la combinación de rastrojo de tarwi con guano, genera mayor productividad.
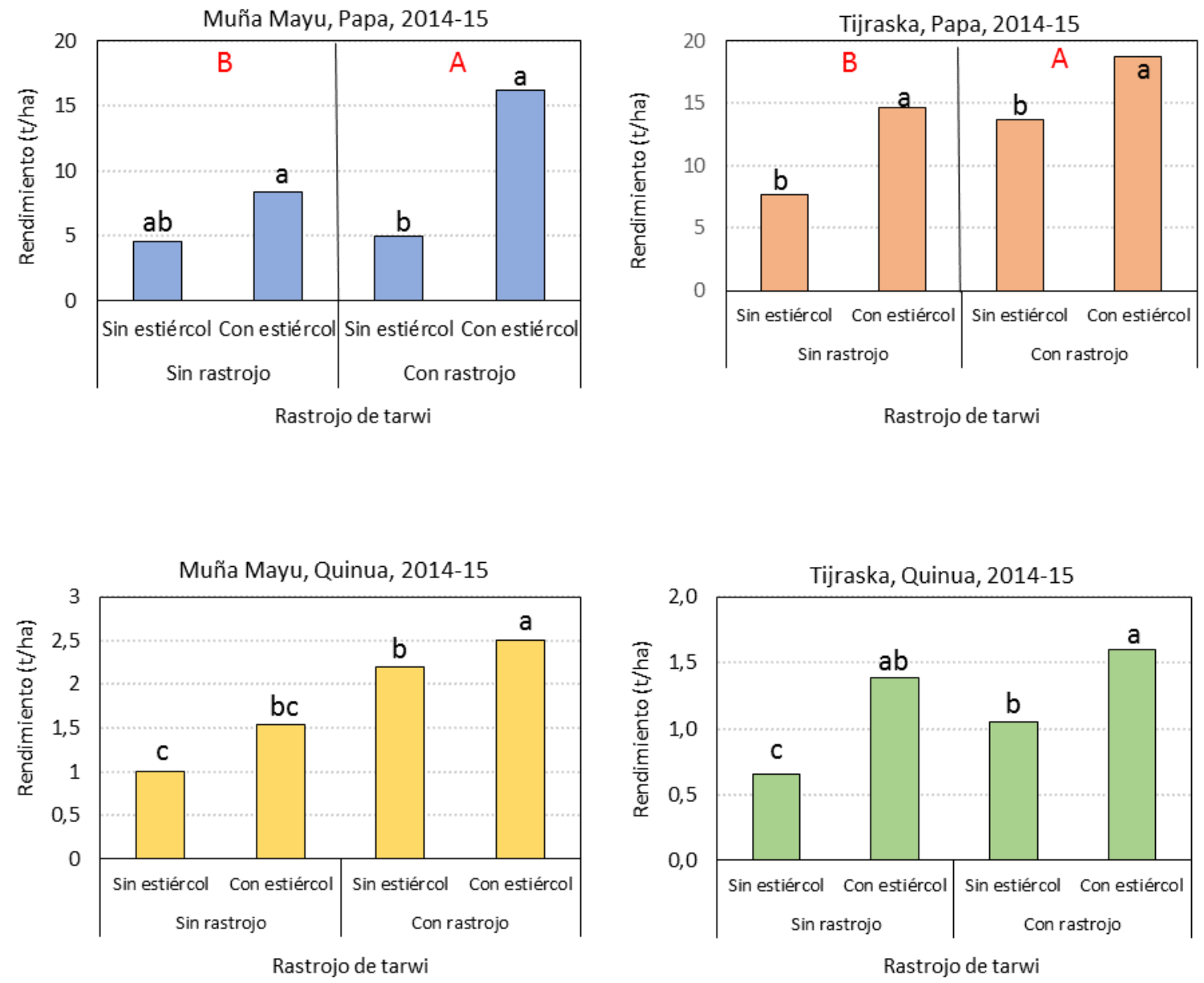

Figura 1. Efecto de la incorporación de rastrojo de tarwi (L. mutabilis) en la productividad de papa y quinua en las ecorregiones "Planicie alta y seca" (Comunidad Muña Mayu) y "Ladera media" (Comunidad Tijraska) de Anzaldo. 2014-2015. Letras iguales son estadísticamente similares al $95 \%$ de probabilidad. 
Revista Latinoamericana de la Papa 22 (2): 55 - 65

http://www.papaslatinas.org/revista.html

Una manera indirecta de medir la actividad microbiana del suelo es medir su respiración. El efecto del rastrojo de tarwi y el guano ovino sobre la respiración del suelo durante el desarrollo de los cultivos de papa y quinua, se muestra en la Figura 2.

Se aprecia mayor respiración en las parcelas donde se incorporó rastrojo de tarwi, y donde éste se combina con guano, respecto a las parcelas sin incorporación de rastrojo de tarwi. Este comportamiento sugiere que la materia orgánica generada por el rastrojo de tarwi, es un buen substrato para generar una mayor actividad microbiana y consecuentemente una mayor respiración del suelo
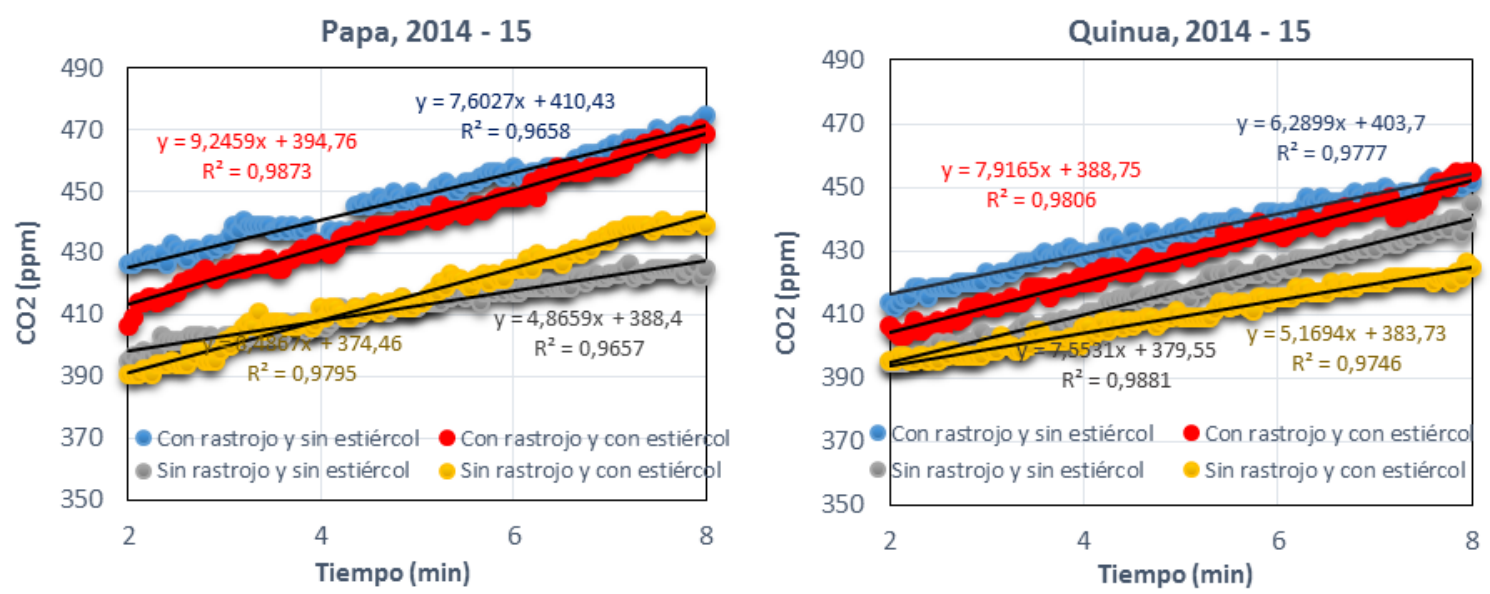

Figura 2. Tasa de respiración del suelo por efecto de la incorporación de residuos de cosecha de tarwi para la producción de papa y quinua. Anzaldo 2014 - 2015.

Para corroborar los anteriores resultados en el cultivo de papa, en la campaña 2015-2016, se repitió el estudio anterior donde se incluyó un nuevo tratamiento, que representaba a aquellas parcelas donde no se cultiva tarwi, a manera de testigo local, es decir un tratamiento SIN tarwi y SIN rastrojo a nivel de parcela principal, siendo un tratamiento que no fue considerado en la campaña anterior (Figura 3). Las letras mayúsculas comparan las medias del factor "rastrojo de tarwi" (parcela principal) mientras que las letras minúsculas comparan las medias del factor "guano ovino" (sub parcela). 


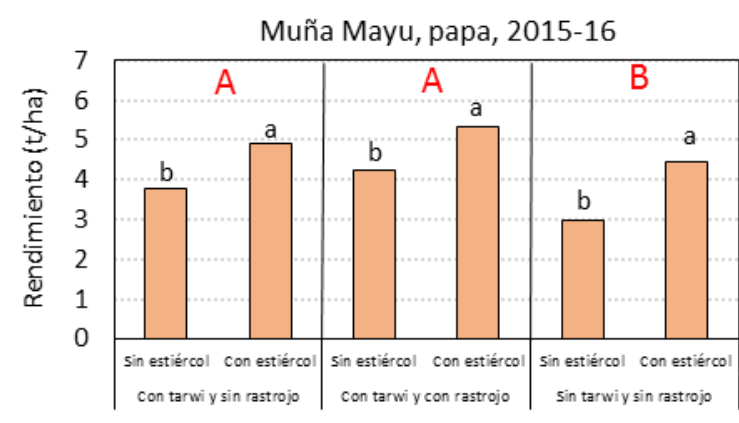

Tarwi y rastrojo

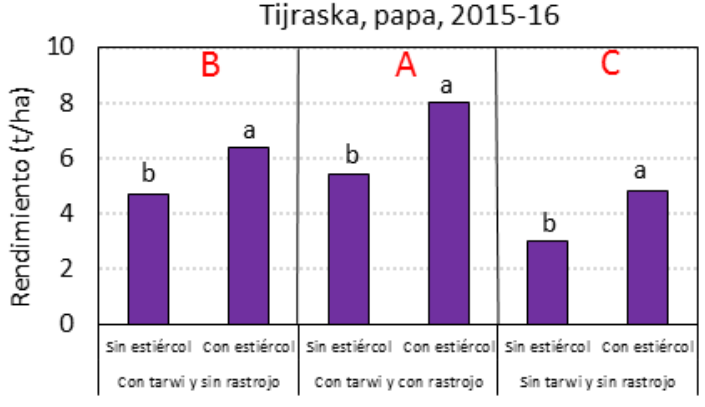

Tarwi y rastrojo

Figura 3. Efecto del cultivo de tarwi (L. mutabilis), de la incorporación de su rastrojo y del guano ovino, en la productividad de papa en las ecorregiones "Planicie alta y seca" (Comunidad Muña Mayu) y "Ladera media" (Comunidad Tijraska) de Anzaldo. 20152016. Letras iguales son estadísticamente similares al 95\% de probabilidad.

Considerando que fue un año agrícola más seco que el anterior, el efecto del rastrojo solo fue perceptible en la eco región 2 (Tijraska) y no así en la ecoregión 1 (Muña Mayu), la cual se caracteriza por tener suelos más superficiales y más proclives a la sequedad, aspecto que probablemente no permitió una adecuada descomposición del rastrojo. Contrariamente, bajo mejores condiciones de suelo como los de Tijraska, pese a la sequía, la descomposición fue mayor, lo que permitió demostrar nuevamente que el rastrojo de tarwi logra mejorar la productividad de la papa (Figura 3), debido a su efecto favorable en el suelo (Figuras 4 y 5).

Esta respuesta del rendimiento, muestra que bajo las condiciones de la eco región 2 (Tijraska), un tiempo de 7 a 8 meses es suficiente para lograr descomponer los residuos de tarwi, a tal punto que sus nutrientes estén a disposición de los cultivos del sistema de rotación. Por otro lado, también se aprecia que el guano logra incrementar la productividad de la papa, y este efecto es mayor cuando se acompaña con rastrojo de tarwi.
En la misma Figura 3 también se aprecia que en las parcelas donde se cultivó tarwi la productividad de papa fue mayor respecto a las parcelas donde no se cultivó, este aspecto muestra el efecto favorable del tarwi en el sistema de rotación, aún si su rastrojo no es incorporado al suelo.

En general, en ambas campañas (2014-15 y 2015-16) se pudo determinar que el efecto residual de la incorporación de rastrojo de tarwi al suelo, mejora la productividad del cultivo de papa $y$ quinua. En ambos cultivos, la combinación de rastrojo de tarwi con guano es el que genera mayor productividad.

El efecto del rastrojo de tarwi y el guano ovino sobre la respiración del suelo, durante el desarrollo de los cultivos de papa, se muestra en la Figura 4. 

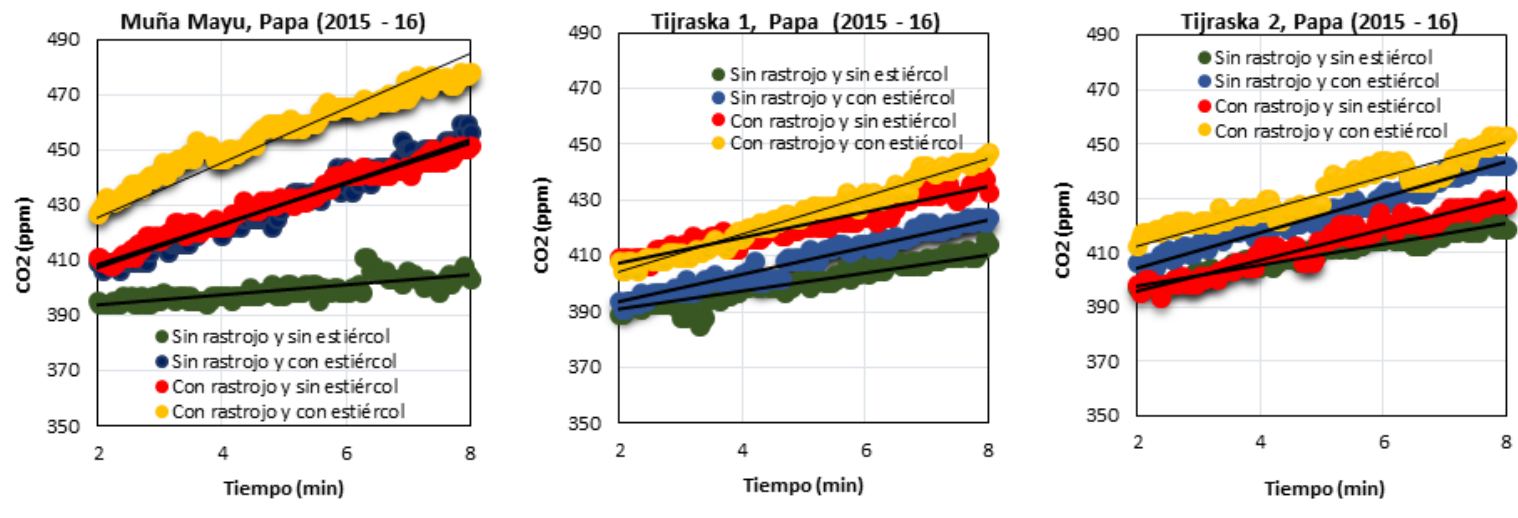

Figura 4. Tasa de respiración del suelo por efecto de la incorporación de residuos de cosecha de tarwi para la producción de papa en las ecorregiones de "Planicie alta y seca" (Comunidad Muña Mayu) y "Ladera media" (Comunidad Tijraska) de Anzaldo. 2015 - 16.

Al igual que los resultados del estudio anterior (Figura 3), las parcelas donde se incorporó rastrojo de tarwi y donde éste se combinó con guano, presentan mayor respiración respecto a las parcelas sin incorporación.

En general, en ambas campañas (2014-15 y 2015-16) se pudo determinar que el rastrojo de tarwi, incorporado al suelo, logra incrementar su respiración lo que sugiere que la materia orgánica generada por el rastrojo, se constituye es un substrato que genera mayor actividad microbiana.

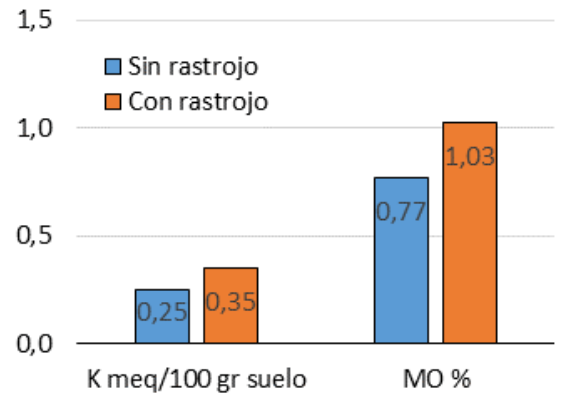

A

Figura 5. Efecto de la incorporación de residuos de cosecha de tarwi en el contenido de potasio y materia orgánica del suelo (A) y en el contenido de nitrógeno o fósforo del suelo (B). Anzaldo 2014 - 2015.
Las condiciones de calor y humedad que se presentaron entre septiembre y febrero, favorecieron la descomposición; aunque, durante el desarrollo de los cultivos, se pudo apreciar pequeñas porciones de rastrojo que no se descompusieron totalmente, porque no estaban incorporadas de la manera más adecuada.

El análisis químico de los suelos (Figura 5) muestra que la incorporación de los residuos de tarwi (rastrojo), logró incrementar el contenido de materia orgánica, nitrógeno (NH4), fósforo disponible y potasio.

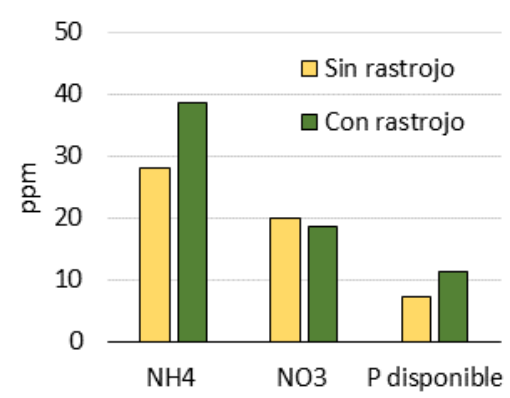


Revista Latinoamericana de la Papa 22 (2): 55 - 65 http://www.papaslatinas.org/revista.html

\section{Agradecimientos}

El presente trabajo de investigación se realizó en el marco de un proyecto financiado por la Fundación McKnight a quien enviamos nuestro agradecimiento porque permitió generar información valiosa para encontrar alternativas tecnológicas que permitan recuperar los suelos marginales de regiones andinas semiaridas de Bolivia, donde la papa está en proceso de erosión debido a la reducción del potencial productivo de los suelos por causas antrópicas y del cambio climático.

\section{Conflicto de intereses}

Los autores declaran que no existen conflictos de intereses.

\section{Referencias citadas}

Barrera, C. (2015). Evaluación del frijol lupinus (Lupinus mutabilis) como abono verde para la producción agroecológica en el municipio de Subachoque Cundinamarca. Tesis Ingeniería en Agroecología. Corporación Universitaria Minuto de Dios. Facultad de Ingeniería. Bogotá, Colombia. 79 p.

Campbell, C.; Conkey, B.; Zentner, R.; Sellers, F.; Curtin, D. (1996). Long term effects of tillage and crop rotations on soil organic $\mathrm{C}$ and total $\mathrm{N}$ in a clay soil in south western Saskatchewan. Canada. J. Soil. Sci.76: 395-401.

Cifuentes, R.; Núñez, E.; Espinosa, H.; Alcántar, G. (2001). Asociación lupinomaíz en la nutrición fosfatada en un Andosol. Terra Latinoamericana. Sociedad Mexicana de la Ciencia del Suelo. Chapingo, México Vol. 19. Nro. 2. pp. 141-154.
Eiza, M.; Fioriti, N.; Studdert, G.; Echeverría, H. (2005). Fracciones de carbono orgánico en la capa arable: efecto de los sistemas de cultivo y fertilización nitrogenada. Ciencia. Suelo. Versión Online. Vol. 23, Nro.1. Buenos Aires, Argentina. pp. 1-12.

Espinoza, Y.; Lozano, Z.; Velásquez, L. (2007). Efecto de la rotación de cultivos y prácticas de labranza sobre las fracciones de la materia orgánica del suelo. Asociación Interciencia. Caracas, Venezuela. Vol. 32. pp. 554-559.

Follet, R. (2001). Soil management concepts and carbon sequestration in crop land soils. Soil Till. Res. 61: 77-92.

Fonte, S.; Vanek, S. (2012). Rutas de intensificación agroecológica para el manejo de la fertilidad del suelo dirigidas a los pequeños agricultores de las zonas alto-andinas. Informe y recomendaciones para la fundación McKnight. 102 p.

Hudson, B. (1994). Soil organic matter and available water capacity. Soil and water conservation Society 49 (2):189194.

Omay, A.; Rice C.; Maddux, L.; Gordon, W. (1997). Changes in soil microbial and chemical properties under long term crop rotation and fertilization. Soil Sci. Soc. Am. J. 61: 1672-1678.

Oros, R.; Lazarte, M.; Alemán, A. (2018). Herramientas metodológicas para analizar la incorporación de nuevos cultivos en sistemas sociales y económicos de alta vulnerabilidad. Revista de Agricultura. Nro. 57. FCAyP-CIF-PROINPA. Cochabamba, Bolivia. En prensa.

Potter, K.; Tolbert, H.; Jones, O.; Matocha, J.; Morrison, J.; Unger, P. (1998). Distribution and amount of soil organic $\mathrm{C}$ in long term management 
system in Texas. Soil Till. Res. 47: 309321.

Spedding, T.; Hamel, C.; Mehuys, G.; Madramootoo, C. (2004). Soil microbial dynamics in maize growing soil under different tillage and residue management systems. Soil Biology and Biochemistry. 36: 499-512.

Studdert, G.; Echeverría, H. (2000). Crop rotations and nitrogen fertilization to manage soil organic carbon dynamics. Soil Science Society of America. Journal Abstract. Vol. 64 No. 4: 1496-1503. 